\title{
Clinical Uses of Diffusion Tensor Imaging Fiber Tracking Merged Neuronavigation with Lesions Adjacent to Corti- cospinal Tract : A Retrospective Cohort Study
}

\author{
Qi Yu, ${ }^{1-3}$ Kun Lin, ${ }^{4}$ Yunhui Liu, ${ }^{1-3}$ Xinxing Li ${ }^{1-3}$ \\ Department of Neurosurgery, Shengjing Hospital of China Medical University, Shenyang, China \\ Liaoning Clinical Medical Research Center in Nervous System Disease, ${ }^{2}$ Liaoning, China \\ Liaoning Key Laboratory of Neuro-Oncology, Liaoning, China \\ Department of Radiology, ${ }^{4}$ Shengjing Hospital of China Medical University, Shenyang, China
}

Objective : To investigate the efficiency of diffusion tensor imaging (DTI) fiber-tracking based neuronavigation and assess its usefulness in the preoperative surgical planning, prognostic prediction, intraoperative course and outcome improvement.

Methods : Seventeen patients with cerebral masses adjacent to corticospinal tract (CST) were given standard magnetic resonance imaging and DTI examination. By incorporation of DTI data, the relation between tumor and adjacent white matter tracts was reconstructed and assessed in the neuronavigation system. Distance from tumor border to CST was measured.

Results : The sub-portion of CST in closest proximity to tumor was found displaced in all patients. The chief disruptive changes were classified as follows : complete interruption, partial interruption, or simple displacement. Partial interruption was evident in seven patients (41.2\%) whose lesions were close to cortex. In the other 10 patients (58.8\%), delineated CSTs were intact but distorted. No complete CST interruption was identified. Overall, the mean distance from resection border to CST was $6.12 \mathrm{~mm}$ (range, $0-21$ ), as opposed to $8.18 \mathrm{~mm}$ (range, 2-21) with simple displacement and $2.33 \mathrm{~mm}$ (range, 0-5) with partial interruption. The clinical outcomes were analyzed in groups stratified by intervening distances (close, $<5 \mathrm{~mm}$; moderated, $5-10 \mathrm{~mm}$; far, $>10 \mathrm{~mm}$ ). For the primary brain tumor patients, the proportion of completely resected tumors increased progressively from close to far grouping $(42.9 \%, 50 \%$, and $100 \%$, respectively). Five patients out of seven $(71.4 \%)$ experienced new neurologic deficits postoperatively in the close group. At meantime, motor deterioration was found in six cases in the close group. All patients in the far and moderate groups received excellent (modified Rankin Scale [mRS] score, $0-1$ ) or good (mRS score, 2-3) rankings, but only $57.1 \%$ of patients in the close group earned good outcome scores.

Conclusion : DTI fiber tracking based neuronavigation has merit in assessing the relation between lesions and adjacent white matter tracts, allowing prediction of patient outcomes based on lesion-CST distance. It has also proven beneficial in formulating surgical strategies.

Key Words : Magnetic resonance imaging · Diffusion tensor imaging $\cdot$ Tractography $\cdot$ Neuronavigation $\cdot$ Tumor.

- Received : February 12, 2019 •Revised : March 27, 2019 •Accepted : April 4, 2019

- Address for reprints : Xinxing Li

Department of Neurosurgery, Shengjing Hospital of China Medical University, 36 Sanhao St. Heping Qu, Shenyang Shi, Liaoning Sheng 110003, China

Tel : +86-24-96615-36111, Fax : +86-24-2295-8989, E-mail : lixx8611@163.com, ORCID : https://orcid.org/0000-0002-8329-3587

This is an Open Access article distributed under the terms of the Creative Commons Attribution Non-Commercial License (http://creativecommons.org/licenses/by-nc/4.0) which permits unrestricted non-commercial use, distribution, and reproduction in any medium, provided the original work is properly cited. 


\section{INTRODUCTION}

Maximizing the surgical resection of tumors in eloquent brain areas while minimizing injury to vulnerable cortex and white matter tracts remains a challenge, given the considerable risk to related corticospinal tracts $(\mathrm{CSTs})^{3)}$. Although microinvasive techniques have drastically improved chances of surgical success $^{8,11,20)}$, white matter tracts may at times sustain injury, culminating in severe neurologic deficits and poor outcomes.

In recent decades, neuronavigation techniques have enabled greater precision in localizing deep-seated brain lesions to ensure surgical accuracy and leave adjacent uninvolved tissues intact ${ }^{28)}$. Such advances clearly have helped enhance patient outcomes. Diffusion tensor imaging (DTI) is a relatively new and ground-breaking technology that makes possible rotationally invariant descriptions of the endowed shape of water diffusion. Invariance to rotation is an innovative feature, enabling application of DTI method to the anatomic complexities of fiber tracts in the human brain ${ }^{31)}$. Accordingly, DTI has proven useful for various neurologic and neurosurgical purposes. By offering a means of exploring plastic/reactive changes in the microstructure and connectivity, study of the human connectome is now both feasible and reproducible ${ }^{4,13,14)}$. Researchers have also acknowledged that DTI has specific estimative value in certain neurodegenerative and neurologic disorders such as Alzheimer's disease ${ }^{10)}$, traumatic brain injury ${ }^{22)}$, and stroke $\mathrm{e}^{7}$. Moreover, the unique capacity of DTI to depict white matter tracts anatomically has particular utility in preoperative brain tumor imaging and surgical planning ${ }^{12)}$.

The present retrospective study reflects our single-center experience using DTI-based neuronavigation as a clinical tool in the treatment of patients with brain tumors. A review of the literature was undertaken as well, focusing on the benefits of this approach in terms of preoperative surgical planning, prognostic prediction, intraoperative surgical strategies, and outcome improvement.

\section{MATERIALS AND METHODS}

\section{Patient data}

Between 2015 and 2018, seventeen patients with lesions close to the crucial cortical lobes (i.e., precentral and postcentral gyrus) or subcortical white matter, all diagnosed through magnetic resonance imaging (MRI), underwent surgeries assisted by DTI fiber tracking-based neuronavigation (BrainLAB, Feldkirchen, Germany) within the department of neurosurgery. Eligible patients were those with newly diagnosed single intracranial lesion situated adjacent to CSTs. Patients with multiple or recurrent intracranial lesions were excluded, as were those whose pre- and postoperative clinical assessment data were incomplete. All the surgeries were performed by the same neurosurgeon. All procedures performed in studies involving human participants were in accordance with the ethical standards of the Ethical Committee of Shengjing Hospital of China Medical University and with the 1964 Helsinki declaration and its later amendments or comparable ethical standards (approval No. 2015064).

\section{Preoperative DTI fiber tracking and neuronavi- gation plans}

Careful neurologic examinations were performed upon admission. To then fully characterize each lesion in terms of adjacent landmarks (i.e., pre- and postcentral gyrus, skull base, and critical cranial nerves and arteries), standard T1and T2-weighted, fluid-attenuated inversion recovery, and T1 contrast-enhanced MRI sequences were obtained via 3.0-Tesla scanner (Ingenia, Philips, Healthcare, Amsterdam, the Netherlands), equipped with an 8-channel head coil (field of view, $220 \times 220 \mathrm{~mm}$; slice thickness, $2.00 \mathrm{~mm}$ ). DTI was performed using a spin echo-echo planar imaging sequence (repetition time, $1677 \mathrm{msec}$; echo time, $83 \mathrm{msec}$; matrix, $144 \times 144$; slice thickness, $2.5 \mathrm{~mm}$; slice gap, $2.5 \mathrm{~mm}$ ). A b=0 image, as well as 36 non-collinear directions of $b=800$ images, were collected and reviewed by one of the senior authors. All DTI data were processed in a Philips Extended MR Workspace (2.6.3.1; Philips, Best, the Netherlands) workstation. Diffusion registration software was engaged to avoid susceptibility artifact and distortion.

The MRI and DTI data (DICOM format) were transferred to the neuronavigation system for computer-driven fiber tracking as described elsewhere ${ }^{25}$, applying proprietary software (BrainLAB iPlan Cranial v3.0.5; BrainLAB). By preliminarily marking every MRI slice, a reconstructed three-dimensional model of each tumor was generated. A sub-portion of CST was then depicted using two regions of interest (ROIs) in motor cortex and ipsilateral brainstem. A default fractional anisotropy (FA) threshold of 0.20 and a minimum fiber length 
of $50 \mathrm{~mm}$ remained constant for each patient, as has been previously reported ${ }^{17)}$. The iteration was started and continued until certain stop criteria, such as FA value and fiber angulation $\left(>30^{\circ}\right)$, were reached ${ }^{16)}$. Finally, the delineated white matter bundle was registered into a T1-weighted MR data set. The distance from tumor to the delineated sub-portion of CST was measured, and the results of the DTI analysis were rigidly registered through anatomical datasets. The accuracy of anatomic registration was carefully reviewed by test-retest visual analysis as reported previously ${ }^{40)}$.

\section{Surgical procedure, MEP monitoring and intra- operative neuronavigation}

To minimize local tissue injury, a neuronavigation system (Cranial 2.1.5; BrainLAB), incorporating consolidated preoperative anatomical images and DTI fiber-tracking data was systematically deployed to design the bone flap and to determine overall areas of lesions upon opening of dura. In all patients, the procedures were microsurgical in nature, performed under general anesthesia using a suitable operative approach (Table 1). Transcranial electrical stimulation technique was conducted as published before ${ }^{34)}$. Nicolet endeavor
CR monitoring system was used. Stimuli were delivered through subdermal needle electrodes placed at C3 and C4 (International 10-20 system of electroencephalogram electrode placement system). Stimuli were delivered in trains of 5 stimuli at $500 \mathrm{~Hz}$; up to $400 \mathrm{~V}$ in intensity. The train duration was $300 \mu$ s. Subdermal needle electrodes were used for recording compound motor action potentials in the target muscles. The fundamental surgical techniques have been described previously ${ }^{19,21,30,32,39)}$. Once the resection commenced, the initial surgical approach was monitored through neuronavigation and modified if CST integrity seemed at risk. When nearing CSTs, tumor margins were double-checked to ensure that crucial subcortical structures and CSTs were well protected. A perioperative lumbar drain was used one instance only to achieve adequate brain relaxation (pterional approach for a large glioblastoma multiforme). Each surgical specimen was subjected to histopathologic assessment.

\section{Postoperative evaluations and outcomes in fol- low-up data collection}

MRI or CT studies were routinely performed 3 days postoperatively to gauge the adequacy of lesion removal. Findings

Table 1. Clinical characteristics of patients

\begin{tabular}{|c|c|c|c|c|c|}
\hline Case No. & Location & $\begin{array}{c}\text { Tumor volume } \\
\left(\mathrm{cm}^{3}\right)\end{array}$ & Clinical manifestation & Surgical approach & Tumor histology \\
\hline 1 & Frontal & 35 & Cognitive changes, memory loss & Transfrontal approach & High grade glioma \\
\hline 2 & Left lateral ventricle & 23 & Headache & Transinferior parietal approach & Meningioma \\
\hline 3 & Temporal & 28 & Headache, seizures & Transtemporal approach & Cavernous malformation \\
\hline 4 & Frontal & 41 & Headache, language deficit & Transpterional approach & Glioblastoma multiforme \\
\hline 5 & Parietal & 19 & Motor impairment & Transparietal approach & Metastatic brain tumor \\
\hline 6 & Parietal & 18 & Motor impairment & Transparietal approach & Metastatic brain tumor \\
\hline 7 & Temporal & 40 & Headache, motor impairment & Transtemporal approach & Low grade glioma \\
\hline 8 & Parietal & 21 & Headache, numbness & Transparietal approach & Cavernous malformation \\
\hline 9 & Temporal & 24 & Headache & Transtemporal approach & Lymphoma \\
\hline 10 & Frontal, temporal & 45 & Headache, motor impairment & Transpterional approach & Glioblastoma multiforme \\
\hline 11 & Temporal & 17 & Headache, seizures & Transtemporal approach & Cavernous malformation \\
\hline 12 & Right lateral ventricle & 25 & Motor impairment & Transinferior parietal approach & High grade glioma \\
\hline 13 & Temporal & 28 & Headache, motor impairment & Transtemporal approach & High grade glioma \\
\hline 14 & Temporal & 21 & Headache & Transtemporal approach & High grade glioma \\
\hline 15 & Parietal & 39 & Motor impairment & Transinferior parietal approach & High grade glioma \\
\hline 16 & Trigonal region & 30 & Headache & Transinferior parietal approach & Glioblastoma multiforme \\
\hline 17 & Parietal & 28 & Language deficit & Transinferior parietal approach & Low grade glioma \\
\hline
\end{tabular}


of neurologic examinations conducted at time of discharge and at first and most recent postoperative visits were also recorded. Functional states of patients were determined independently by two individual observers at time of admission, at discharge, and at the 3-month postoperative outpatient clinic visit. The modified Rankin Scale (mRS) was applied (excellent, 0-1; good, 2-3; bad, 4-6) to assess patient status preoperatively and at outpatient clinic visits.

\section{Statistical analysis}

A descriptive analysis of patient characteristics, including related measures and clinical assessments, was conducted using standard software (SPSS ver. 16.0; SPSS Inc., Chicago, IL, USA). Absolute or relative frequencies of qualitative variables were expressed as percentages and quantitative variables as means or ranges.

\section{RESULTS}

\section{Patient population and tumor characteristics}

Nine men (52.9\%) and eight women (47.1\%) were selected for study, ranging from 29-72 years of age (mean, 53.2 years).
Lesion volume ranged from 17-45 $\mathrm{cm}^{3}$ (mean, $28.3 \mathrm{~cm}^{3}$ ). Clinical manifestations and lesion locations are presented in Table 1.

\section{Histopathological diagnosis}

Histopathologic studies ultimately confirmed 10 primary brain tumors including three glioblastoma multiformes, five high grade gliomas (World Health Organization [WHO] grade III-IV), two low grade gliomas (WHO grade I-II), three cavernous malformations, two metastatic brain tumors, one meningioma, and one lymphoma.

\section{Preoperative DTI fiber tracking}

The mean time from DTI data acquisition to operative intervention was 7.5 days. As shown in Fig. 1A, tractography profiles were generated via color-coded FA-map. Counterpart CSTs in hemispheres contralateral to those of respective tumors served as normal controls. Due to space-occupying effect and surrounding edema, the sub-portion of CST in closest proximity to tumor was found displaced in all patients. If the volume of the counterpart CSTs with the same ROI was taken as a normal control, chief disruptive changes were classified according to the integrity of delineated CST in the
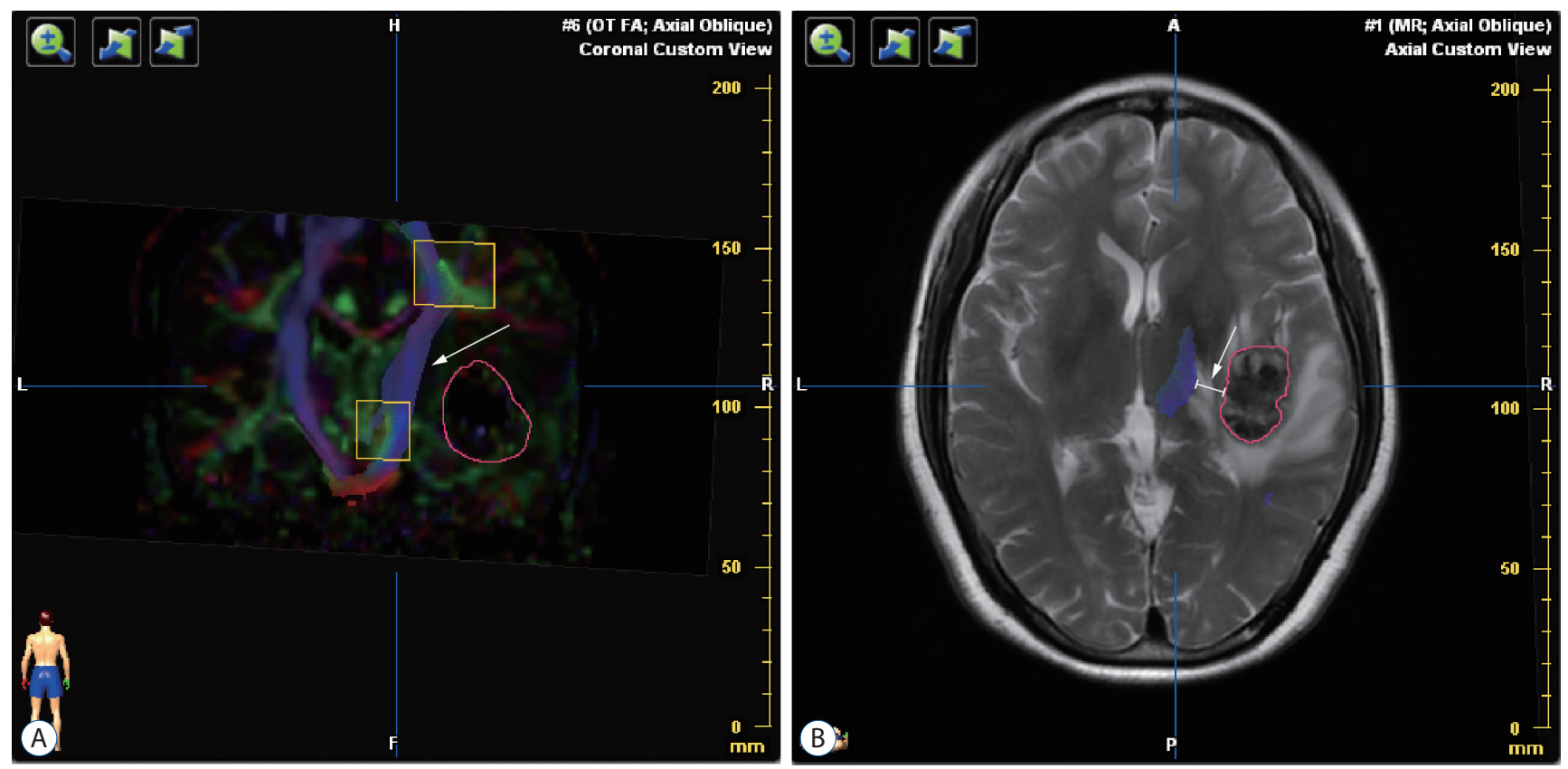

Fig. 1. Preoperative diffusion tensor imaging fiber tracking. A : Tractography was assessed based on color-code fractional anisotropy map. The sub-portion of right corticospinal tract was intact but interiorly distorted (white arrow) by the lateral tumor (the red circle). B : The distance from tumor border to corticospinal tract was measured in each patient (white arrow). 
lesion hemisphere: complete interruption (CST was not displayed), partial interruption (partial bundle of CST was displayed), or simple displacement (partial bundle of CST was displayed). Partial interruption was evident in seven patients (41.2\%) whose lesions were close to cortex. In the other 10 patients (58.8\%), delineated CSTs were intact but distorted. The lesions in these patients were situated near cerebral peduncle. No complete CST interruption was identified. Overall, the distance from resection border to CST was measured in the fusion image (Fig. 1B) with a mean of 6.12 $\mathrm{mm}$ (range, 0-21), as opposed to $8.18 \mathrm{~mm}$ (range, 2-21) with simple displacement and $2.33 \mathrm{~mm}$ (range, $0-5$ ) with partial interruption (Table 2).

\section{Intraoperative modification of surgical approach}

All patients underwent neuronavigation-assisted lesion removal, cross-referencing DTI results with planned surgical tactics. The skull flaps were designed to accommodate respective lesions, the locations of which were double-checked preoperatively. In 13 patients $(76.5 \%)$ whose tumors were radiographically lateral to the white matter tracts, surgery was executed as originally planned. Surgical approach was modified intraoperatively in four patients (23.5\%), each displaying lateral CST distortion (in cortical direction) and thus a greater risk of CST injury. One of the patients was planned to undergo tumor removal through anterior transcallosum approach. After DTI and neuronavigation analysis, we found it's hard to get the tumor gross resection and then performed a tumor resection through transinferior parietal approach. Seven patients (41.2\%) showed pial presentation at preoperative radiologic examination, reliably indicative of a resection plane requiring minimal normal brain tissue retraction. In 10 patients (58.8\%) without clear evidence of pial presentation by preoperative MRI, the decisions were based on results of T2-weighted and DTI data.

\section{Illustrative case 1}

A 29-year-old woman presented to our service, having experienced a seizure and subsequent severe headache. Preoperative MRI revealed a cavernous malformation of right temporal lobe, with pial presentation (Fig. 2A). DTI fiber tracking

Table 2. Preoperative findings and postoperative results

\begin{tabular}{|c|c|c|c|c|c|c|c|c|c|}
\hline \multirow{2}{*}{ Case No. } & \multirow{2}{*}{$\begin{array}{l}\text { Distance* } \\
(\mathrm{mm})\end{array}$} & \multirow{2}{*}{ CST changes } & \multirow{2}{*}{$\begin{array}{l}\text { Adequacy of } \\
\text { resection }\end{array}$} & \multirow{2}{*}{$\begin{array}{l}\text { Neurological } \\
\text { deficits }\end{array}$} & \multirow{2}{*}{$\begin{array}{l}\text { Follow up } \\
\text { (months) }\end{array}$} & \multicolumn{2}{|c|}{ Motor function } & \multicolumn{2}{|c|}{$\mathrm{mRS}$} \\
\hline & & & & & & Preop & Postop & Preop & Postop \\
\hline 1 & 13 & SD & Complete & NA & 6 & 4 & 5 & 1 & 0 \\
\hline 2 & 21 & SD & Complete & NA & 18 & 5 & 5 & 1 & 0 \\
\hline 3 & 10 & SD & Complete & NA & 22 & 4 & 5 & 2 & 0 \\
\hline 4 & 5 & PI & Incomplete & Temporary & 6 & 3 & 3 & 3 & 3 \\
\hline 5 & 2 & SD & Complete & NA & 4 & 4 & 3 & 3 & 2 \\
\hline 6 & 3 & $\mathrm{PI}$ & Complete & Temporary & 3 & 4 & 3 & 3 & 2 \\
\hline 7 & 3 & $\mathrm{Pl}$ & Incomplete & Permanent & 9 & 3 & 1 & 3 & 4 \\
\hline 8 & 6 & SD & Complete & NA & 16 & 5 & 5 & 3 & 0 \\
\hline 9 & 11 & SD & Complete & NA & 4 & 5 & 4 & 2 & 1 \\
\hline 10 & 0 & $\mathrm{Pl}$ & Incomplete & Permanent & 4 & 3 & 1 & 3 & 4 \\
\hline 11 & 15 & SD & Complete & NA & 14 & 5 & 5 & 2 & 0 \\
\hline 12 & 0 & SD & Complete & Temporary & 7 & 4 & 3 & 2 & 2 \\
\hline 13 & 2 & $\mathrm{Pl}$ & Incomplete & Permanent & 3 & 3 & 1 & 2 & 4 \\
\hline 14 & 5 & SD & Complete & NA & 9 & 5 & 4 & 2 & 1 \\
\hline 15 & 3 & SD & Complete & NA & 11 & 5 & 4 & 2 & 2 \\
\hline 16 & 1 & $\mathrm{Pl}$ & Incomplete & Temporary & 5 & 4 & 3 & 2 & 3 \\
\hline 17 & 4 & SD & Complete & NA & 6 & 4 & 4 & 3 & 2 \\
\hline
\end{tabular}

*Distance from lesion border to CST. CST : corticospinal tract, mRS : modified Rankin Scale, Preop : pre-operation, Postop : post-operation, SD : simple displacement, NA : not available, PI : partial interruption 

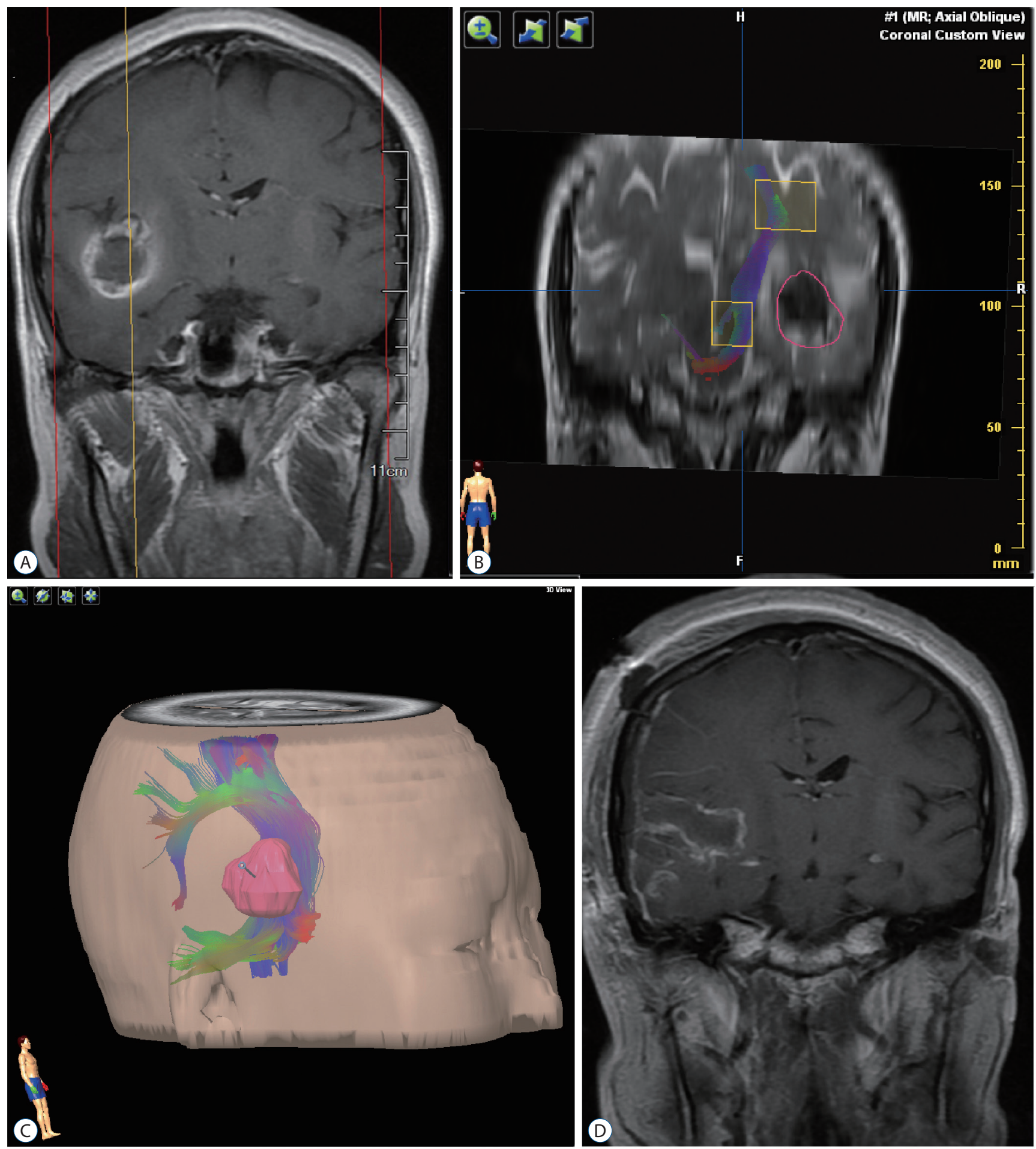

Fig. 2. Illustrative case 1. A 29-year-old woman presented to our service, having experienced a seizure and subsequent severe headache. A : Preoperative magnetic resonance imaging revealed a cavernous malformation of right temporal lobe, with pial presentation. $B$ : Diffusion tensor imaging fiber tracking indicated an intact corticospinal tract, albeit distorted along the central aspect of lesion; and there was a 10-mm distance from presumptive pial border to corticospinal tract. The red circle delineated the tumor border. C: The relation between tumor and corticospinal tract was defined, using our neuronavigation system for surgical trajectory planning. D: Postoperative magnetic resonance imaging showed complete eradication of the lesion. 

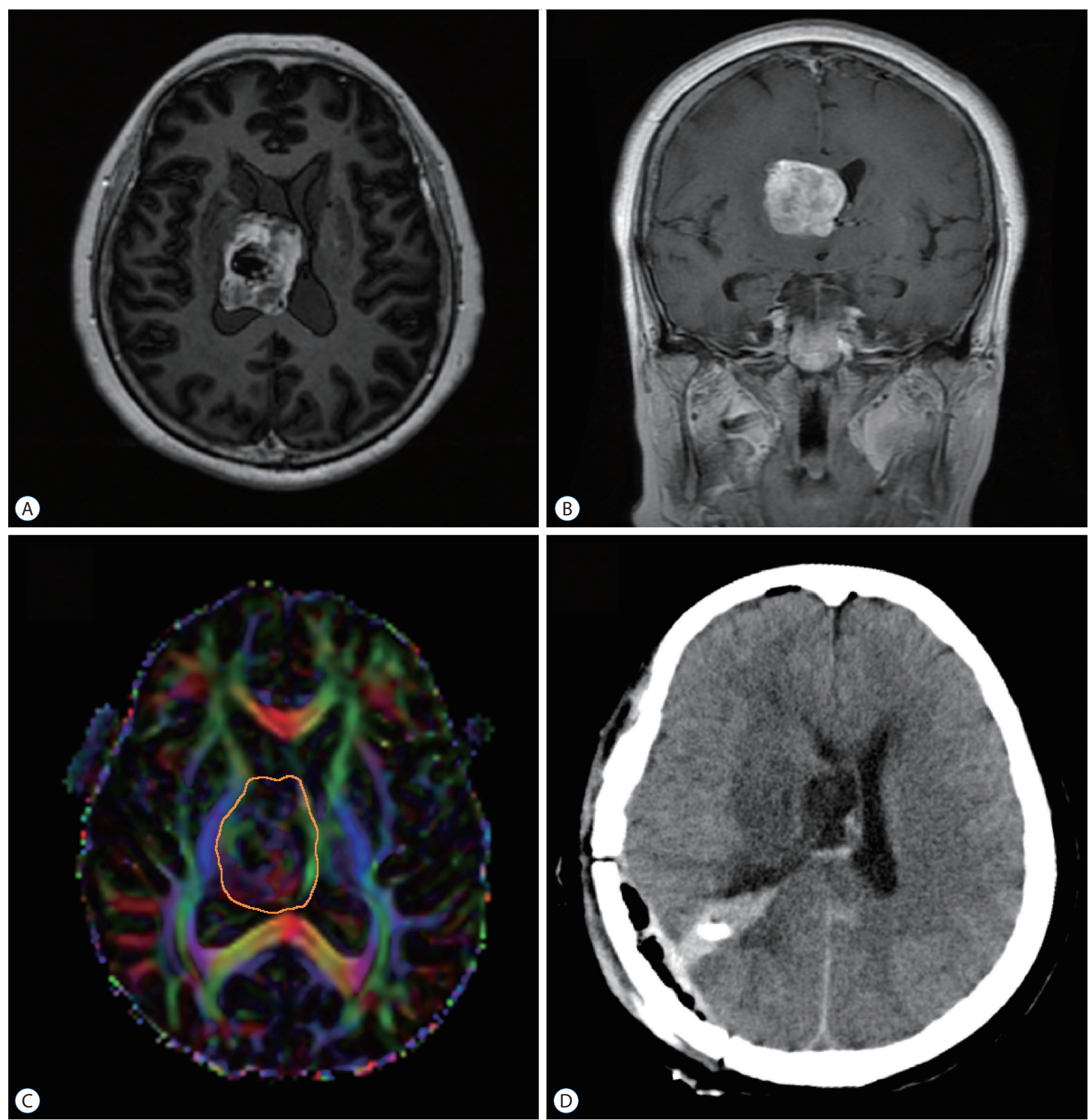

Fig. 3. Illustrative case 2. The patient was a 67-year-old woman who complained about left leg weakness accompanied with blurred vision in both eyes for 2 months. A and B: Preoperative magnetic resonance imaging revealed a large mass situated in the right ventricle. C: DTI analysis showed that the tumor was closely located inside the CST. The tumor-CST was $0 \mathrm{~mm}$ (C). Comparing to the CST in the counterpart hemisphere, the integrity of CST bundle was intact but distorted laterally (simple displacement). The orange circle delineated the tumor border. D : After DTI and neuronavigation analysis, we performed a tumor resection through transinferior parietal approach. The tumor was completely removed under the neuronavigation guidance. DTI: diffusion tensor imaging, CST : corticospinal tract.

indicated an intact CST, albeit distorted along the central aspect of lesion; and there was a $10 \mathrm{~mm}$ distance from presumptive pial border to CST (Fig. 2B). The relation between tumor and CST was defined, using our neuronavigation system for surgical trajectory planning (Fig. 2C). Complete resection was then performed via transcortical temporal approach (as antic- 

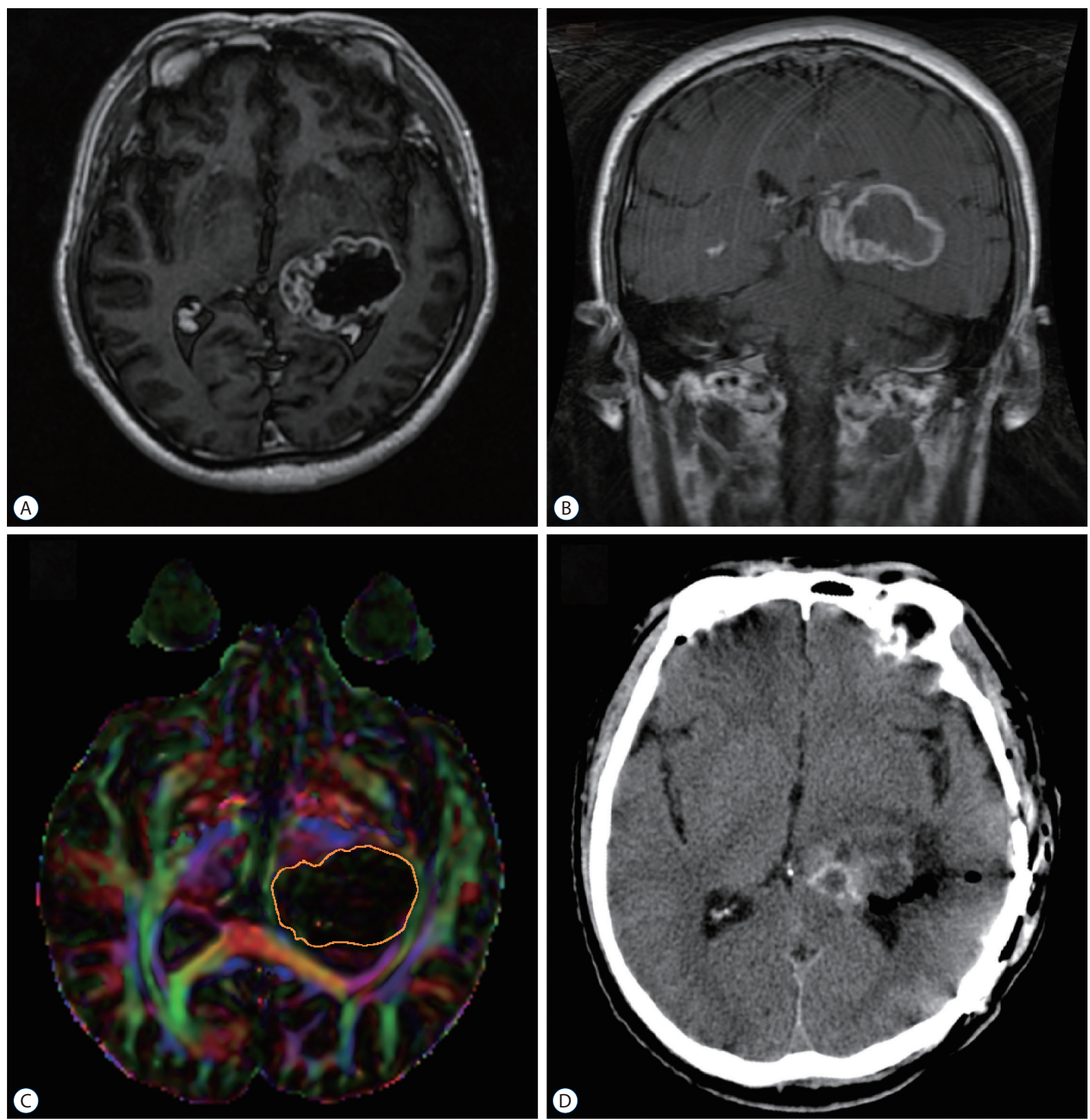

Fig. 4. Illustrative case 3. A 52-year-old man presented with headache and blurred vision for three months. A and B: Preoperative magnetic resonance imaging identified a large tumor at the trigone of lateral. C: DTI analysis revealed that the tumor was closely next to the CST. The tumor-CST distance was 0 mm. Comparing to the CST in the counterpart hemisphere, the integrity of CST bundle was incomplete and distorted anteriorly (partial interruption). The orange circle delineated the tumor border. D : The patient received rumor resection through transinferior parietal approach under the monitor of neuronavigation. DTI : diffusion tensor imaging, CST : corticospinal tract.

ipated) under intraoperative neuronavigation guidance. Both size and location of the skull flap were optimized for this resection. Postoperative MRI showed complete eradication of the lesion (Fig. 2D), and the patient remained free of new neurologic symptoms thereafter. 


\section{Illustrative case 2}

The patient was a 67-year-old woman who complained about left leg weakness accompanied with blurred vision in both eyes for two months. Preoperative MRI revealed a large mass situated in the right ventricle. (Fig. 3A and B). DTI analysis showed that the tumor was closely located inside the CST. The tumor-CST distance was $0 \mathrm{~mm}$ (Fig. 3C). Comparing to the CST in the counterpart hemisphere, the integrity of CST bundle was intact but distorted laterally (simple displacement). The patient was planned to undergo tumor removal through anterior transcallosum approach. After DTI and neuronavigation analysis, we performed a tumor resection through transinferior parietal approach. The tumor was completely removed under the neuronavigation guidance (Fig. 3D). The patient underwent temporally motor deficit postoperatively and recovered to normal after three-month rehabilitation. The histopathology was confirmed high grade glioma (WHO grade III).

\section{Illustrative case 3}

A 52-year-old man presented with headache and blurred vision for three months. Preoperative MRI identified a large tumor at the trigone of lateral ventricle (Fig. 4A and B). DTI analysis revealed that the tumor was closely next to the CST. The tumor-CST distance was $0 \mathrm{~mm}$. Comparing to the CST in the counterpart hemisphere, the integrity of CST bundle was incomplete and distorted anteriorly (partial interruption) (Fig. 4C). The patient received rumor resection through transinferior parietal approach under the monitor of neuronavigation. During the operation, the CST was found at the high risk of injury so that the tumor was incompletely as a result (Fig. $4 \mathrm{D})$. The patient had temporally motor deterioration postoperatively but recovered at the clinic visit five months later. The histopathology was confirmed glioblastoma multiforme.

\section{Postoperative results}

It has been reported that factors that could affect motor deterioration postoperatively included distance between lesion and CST, FA, tumor histopathology, perilesional edema, and tumor volume, etc ${ }^{23)}$. The proximity of lesions to clinically eloquent white matter tracts is regarded the most important factor in postoperative outcomes ${ }^{43)}$. A closeness to the CST usually resulted in a direct damage to CST which will cause a decreased FA. Other factors regarding as perilesional edema or tumor volume are highly related to histopathology. Thus,

Table 3. Comparison of lesion-CST distance* subsets

\begin{tabular}{|c|c|c|c|}
\hline \multirow{2}{*}{ Variable } & \multicolumn{3}{|c|}{ Distance* } \\
\hline & Close $(<5 \mathrm{~mm})$ & Moderate $(5-10 \mathrm{~mm})$ & $\operatorname{Far}(>10 \mathrm{~mm})$ \\
\hline \multicolumn{4}{|l|}{ Primary brain tumor } \\
\hline Complete resection & $3 / 7(42.9)$ & $1 / 2(50.0)$ & $1 / 1(100.0)$ \\
\hline Incomplete resection & $4 / 7(57.1)$ & $1 / 2(50.0)$ & $0 / 1$ \\
\hline Neurological deficits & $5 / 7(71.4)$ & $1 / 2(50.0)$ & $0 / 1$ \\
\hline Motor deterioration & $6 / 7(85.7)$ & $1 / 2(50.0)$ & 0 \\
\hline Excellent (mRS score 0-1) & $0 / 7$ & $1 / 2(66.7)$ & $1 / 1(100.0)$ \\
\hline Good (mRS score 2-3) & $4 / 7(57.1)$ & $1 / 2(33.3)$ & $0 / 1$ \\
\hline Bad (mRS score 4-6) & $3 / 7(42.9)$ & $0 / 2$ & $0 / 1$ \\
\hline \multicolumn{4}{|l|}{ Other lesions } \\
\hline Complete resection & $2 / 2(100.0)$ & $2 / 2(100.0)$ & $3 / 3(100.0)$ \\
\hline Incomplete resection & $0 / 2$ & $0 / 2$ & $0 / 3$ \\
\hline Neurological deficits & $1 / 2(50.0)$ & $0 / 2$ & $0 / 3$ \\
\hline Motor deterioration & $2 / 2(100.0)$ & $0 / 2$ & $1 / 3(33.3)$ \\
\hline Excellent (mRS score 0-1) & $0 / 2$ & $2 / 2(100.0)$ & $3 / 3(100.0)$ \\
\hline Good (mRS score 2-3) & $2 / 2(100.0)$ & $0 / 2$ & $0 / 3$ \\
\hline Bad (mRS score 4-6) & $0 / 2$ & $0 / 2$ & $0 / 3$ \\
\hline
\end{tabular}

Values are presented as number (\%). ${ }^{*}$ Distance from lesion border to CST. CST : corticospinal tract, mRS : modified Rankin Scale 
our patients were stratified by intervening distances (close, $<5$ $\mathrm{mm}$; moderated, 5-10 mm; far, $>10 \mathrm{~mm}$ ). As shown in Table 3, for primary brain tumor patients, the proportion of completely resected tumors increased progressively from close to far grouping (42.9\%, 50\%, and 100\%, respectively). Resections were incomplete in four patients $(57.1 \%)$ in the close group. These tumors were so large that internal segments acting to displace ipsilateral CST or impinging on thalamus was compressed. Additional chemotherapy and radiotherapy were administered, considering the potential for recurrence. Five patients out of seven (71.4\%) experienced new neurologic deficits postoperatively in the close group. In two patients, these deficits proved temporary, each recovering after inpatient rehabilitation. The other three patients sustained permanent new motor deficits. At meantime, motor deterioration was found in six cases in the close group. All patients in the far and moderate groups received excellent ( $\mathrm{mRS}$ score, $0-1$ ) or good (mRS score, 2-3) rankings, but only $57.1 \%$ of patients in the close group earned good outcome scores. For other cerebral lesion patients, resections were complete in all patients. Only one patient received neurological deficits and two patients with motor deterioration in the close group postoperatively. Ranking of mRS score are all good or excellent in these patients. The mean postoperative follow-up duration was 8.65 months. There were no deaths recorded as of the most recent clinic visit. One patient (5.9\%) developed postoperative cerebrospinal fluid leakage, without need of surgical revision. This complication resolved uneventfully after lumbar drain placement.

\section{DISCUSSION}

DTI is an MRI technique enabling detection of water molecules in tissue $\mathrm{e}^{38,42)}$. Its use for neuroimaging was first proposed by Filler et al.9) in 1991. Unlike the random movement observed in the isotropic conditions, water molecules travel along neuronal axons anisotropically, conferring visibility to specific white matter tracts of the central nervous system. Because of its unique characteristics, DTI has found wide usage in research and clinical applications involving physiologic or pathologic states ${ }^{1,2,15)}$.

Reported herein is a retrospective assessment of our singlecenter experience in combining DTI fiber-tracking technique with neuronavigation to surgically manage tumors in vicinities of CSTs. This approach enables the delineation of CSTs, yielding valuable information for surgical planning. Those patients with tumors furthest separated from CSTs fared better clinically than those whose tumors closely approximated CSTs, suggesting that tumor-CST distance may be a valid prognostic index. In addition, DTI data contributed to our surgical strategies, which could be modified on demand in instance of CST distortion to lessen the extent of pyramid pathway injury.

\section{Precise anatomical assessment}

The precise positioning of CSTs is one of the most important determinants of neurosurgical rationale in this setting. Prior to the development of DTI and fiber-tracking technology, the only resource was to estimate CST bearings using anatomical landmarks at tumor perimeters. There are diverse factors associated with degree and pattern of CST displacement or interruption. Tumor-CST distance and tumor location are the most relevant factors pertaining to tractography. As our data have shown, a CST is more apt to be partially or completely interrupted by a tumor in close apposition, given its direct space-occupying effect. Furthermore, tumors in regions near cortex seem inclined to partial CST interruption. The radial contours of subcortical CSTs may be implicated in this regard, complicating the seeding ROI definition ${ }^{41)}$ and performance of surgical procedures. CST impingement may vary as well according to malignancy type. It has been determined DTI tractography is more readily impacted by high-grade gliomas or metastatic tumors through which tract infiltration is aggressively promoted, leading to worse postsurgical neurologic outcomes ${ }^{45)}$. Among the four patients with partial CST interruption that we studied, three presented with high-grade gliomas or metastatic tumors, and only one harbored a lowgrade glioma. CST disruption may also result from edematous tumor peripheries. Whether displaced or not, CSTs are largely undecipherable in the midst of extensive edema but may be verified through stimulation ${ }^{24)}$.

\section{Accuracy determination}

Although white matter tracts are amenable to DTI fiber tracking, the technical validity is still in question ${ }^{6}$, based on inherent limitations or intraoperative shifting after tumor removal. It has been reported that maximum shifting of white matter tracts ranges from -8 to $+15 \mathrm{~mm}^{27)}$. Intraoperative elec- 
trical stimulation is the golden standard for mapping of motor cortex and functional motor tracts ${ }^{5}$, but this was not applied in the present study for various equipment-related reasons. In the absence of intraoperative electrical stimulation, we were unconvinced of the seeding ROI at the precise locations. Nevertheless, all seeding ROIs were defined according to the clinical manifestations of patients incorporated with the motor homunculus in the neuronavigation system. Other researchers have argued that the sole utility of DTI fiber tracking is its precision in reflecting CST pathways ${ }^{43)}$. As a matter of safety, we routinely added 3-5 $\mathrm{mm}$ to CST boundaries in the neuronavigation process. Subsequently, there were no accidental intraoperative CST injuries, and the data on tracked CSTs proved reliable.

\section{Improvement in outcomes}

Because CSTs may be distorted or displaced by the tumors in any direction, accidental injuries when approaching CSTs are potentially avoidable by exercising careful operative technique under DTI navigation. Indeed, preservation of CSTs is critical postoperative outcomes. When examining surgical resections of insular gliomas in 61 patients, Panigrahi et al. ${ }^{29)}$ found that the proportion of patients with normal outcomes at 3 months postoperatively was only $70.5 \%$ in control subjects (DTI not used), compared with 100\% in their DTI group. Similarly, all 11 patients of ours showed improved neurologic function at the 3-months follow-up mark. On occasion, however, incomplete tumor resection was required to preserve motor function, especially if CSTs were infiltrated by tumor ${ }^{26)}$. Thus, the adequacy of resection was diminished. In an earlier report, complete resection was achieved in 79\% of patients through DTI-based neuronavigation, compared with $92 \%$ via intraoperative $\mathrm{MRI}^{2}$. Our complete resection rate was $81.8 \%$, which is aligned with other reported data.

According to our DTI analysis, those patients with tumors furthest from CSTs registered a higher complete resection rate, a lower incidence of postoperative neurologic deterioration, and better outcomes than patients whose tumors closely approximated CSTs. We concluded that a distance of $10 \mathrm{~mm}$ between tumor border and CST was sufficient to preserve motor function. Comparable results were also cited in some prior studies ${ }^{33,35,37)}$. The tumor-CST distance may well be regarded as presumptive predictor of outcome, attributable in part to tumor edema.

\section{Benefit in surgical strategies formation}

In various publications, a multiplicity of surgical strategies has been elected for tumors located adjacent to CSTs, including : transcortical transventricular approach, anterior interhemispheric transcallosal approach, contralateral infratentorial supracerebellar approach, posterior interhemispheric parasplenial approach, and transsylvian transinsular approaches. Each tactic affords tumor access from a specific orientation (anterior, lateral, or posterior). At times, CSTs may reside lateral to the trajectory, making the tumor unreachable without CST dissection or retraction. DTI fiber-tracking may then offer the patient a better approach. Planned surgical approaches were modified in three of our patients, based on DTI-determined lateral distortion or displacement of CSTs.

More studies are increasingly focused on DTI fiber-tracking application for selective nerve fiber protection beyond eloquent brain areas. Zhang et al. ${ }^{44)}$ have utilized DTI fibertracking in the surgical treatment of vestibular schwannomas, proving that tracking modifications are useful for preoperatively predicting tumor location and intraoperatively protecting the facial nerve. Still other researchers are implementing DTI technique in the cortex of spinal cord injuries or tumors $^{18,36)}$. The present study involved a single-center investigation of few patients, contributing largely univariate statistical support. Controlled randomized studies on a broader scale are awaited to confirm the clinical applicability and consistency of our findings.

\section{CONCLUSION}

The neuronavigation system incorporating DTI fiber tracking has merit in assessing the relation between lesions and adjacent white matter tracts, allowing prediction of patient outcomes based on lesion-CST distance. It has also proven beneficial in formulating surgical strategies.

\section{CONFLICTS OF INTEREST}

No potential conflict of interest relevant to this article was reported. 


\section{INFORMED CONSENT}

Informed consent was obtained from all individual participants included in this study.

\section{AUTHOR CONTRIBUTIONS}

\author{
Conceptualization : QY, XL \\ Data curation : KL \\ Formal analysis : QY, XL \\ Funding acquisition : $\mathrm{XL}$ \\ Methodology : KL, YL \\ Project administration : XL \\ Visualization : QY \\ Writing - original draft : QY \\ Writing - review \& editing : XL
}

\section{ORCID}
Qi Yu
https://orcid.org/0000-0003-4026-5356
Kun Lin
https://orcid.org/0000-0002-0011-8258
Yunhui Liu
https://orcid.org/0000-0002-6516-6568
Xinxing Li
https://orcid.org/0000-0002-8329-3587

\section{- Acknowledgements}

The study was supported by the project of "Silybin Induced Autophagy of Glioma Stem Cells to Play An Anti-Tumor Molecular Mechanism" of Key Research and Development Project in Liaoning Province (Grant Number : 2018225094).

\section{References}

1. Andersen $\mathrm{O}$, Hildeman A, Longfils $\mathrm{M}$, Tedeholm H, Skoog B, Tian W, et al. : Diffusion tensor imaging in multiple sclerosis at different final outcomes. Acta Neurol Scand 137 : 165-173, 2017

2. Barone $D G$, Lawrie $T A$, Hart MG : Image guided surgery for the resection of brain tumours. Cochrane Database Syst Rev (1) : CD009685, 2014

3. Benveniste R, Germano IM : Evaluation of factors predicting accurate resection of high-grade gliomas by using frameless image-guided stereotactic guidance. Neurosurg Focus 14 : e5, 2003

4. Bonilha L, Gleichgerrcht E, Fridriksson J, Rorden C, Breedlove JL,
Nesland T, et al. : Reproducibility of the structural brain connectome derived from diffusion tensor imaging. PLoS One 10 : e0135247, 2015

5. Bucci M, Mandelli ML, Berman JI, Amirbekian B, Nguyen C, Berger MS, et al. : Quantifying diffusion MRI tractography of the corticospinal tract in brain tumors with deterministic and probabilistic methods. Neuroimage Clin 3 : 361-368, 2013

6. Bürgel U, Mädler B, Honey CR, Thron A, Gilsbach J, Coenen VA : Fiber tracking with distinct software tools results in a clear diversity in anatomical fiber tract portrayal. Cent Eur Neurosurg 70 : 27-35, 2009

7. Chen Z, Ni P, Zhang J, Ye Y, Xiao H, Qian G, et al. : Evaluating ischemic stroke with diffusion tensor imaging. Neurol Res 30 : 720-726, 2008

8. Di X, Sui A, Hakim R, Wang M, Warnke JP : Endoscopic minimally invasive neurosurgery: emerging techniques and expanding role through an extensive review of the literature and our own experience - part l: intraendoscopic neurosurgery. Pediatr Neurosurg 47 : 315-326, 2011

9. Filler $A G$, Howe FA, Hayes $C E$, Kliot M, Winn HR, Bell BA, et al. : Magnetic resonance neurography. Lancet 341 : 659-661, 1993

10. Fu JL, Liu Y, Li YM, Chang C, Li WB : Use of diffusion tensor imaging for evaluating changes in the microstructural integrity of white matter over 3 years in patients with amnesic-type mild cognitive impairment converting to Alzheimer's disease. J Neuroimaging 24 : 343-348, 2014

11. Garrett $M$, Consiglieri $G$, Nakaji $P$ : Transcranial minimally invasive neurosurgery for tumors. Neurosurg Clin N Am 21 : 595-605, 2010

12. Golby AJ, Kindlmann G, Norton I, Yarmarkovich A, Pieper S, Kikinis R : Interactive diffusion tensor tractography visualization for neurosurgical planning. Neurosurgery 68 : 496-505, 2011

13. Gong G, He Y, Concha L, Lebel C, Gross DW, Evans AC, et al. : Mapping anatomical connectivity patterns of human cerebral cortex using in vivo diffusion tensor imaging tractography. Cereb Cortex 19 : 524-536, 2009

14. Hagmann P, Kurant M, Gigandet X, Thiran P, Wedeen VJ, Meuli R, et al. : Mapping human whole-brain structural networks with diffusion MRI.

PLoS One 2 : e597, 2007

15. Handsfield GG, Bolsterlee B, Inouye JM, Herbert RD, Besier TF, Fernandez JW : Determining skeletal muscle architecture with Laplacian simulations: a comparison with diffusion tensor imaging. Biomech Model Mechanobiol 16 : 1845-1855, 2017

16. Krieg SM, Buchmann NH, Gempt J, Shiban E, Meyer B, Ringel F : Diffusion tensor imaging fiber tracking using navigated brain stimulation--a feasibility study. Acta Neurochir (Wien) 154 : 555-563, 2012

17. Kunimatsu A, Aoki S, Masutani Y, Abe O, Hayashi N, Mori H, et al. : The optimal trackability threshold of fractional anisotropy for diffusion tensor tractography of the corticospinal tract. Magn Reson Med Sci 3 : $11-17,2004$

18. Liu X, Tian W, Chen H, LoStracco TA, Zhang J, Li MY, et al. : Advanced neuroimaging in the evaluation of spinal cord tumors and tumor mimics: diffusion tensor and perfusion-weighted imaging. Semin Ultrasound CT MR 38 : 163-175, 2017

19. Ma J, Cheng L, Wang G, Lin S : Surgical management of meningioma of the trigone area of the lateral ventricle. World Neurosurg 82 : 757769,2014 
20. Manjila S, Karhade A, Phi JH, Scott RM, Smith ER : Real-time ultrasoundguided catheter navigation for approaching deep-seated brain lesions: role of intraoperative neurosonography with and without fusion with magnetic resonance imaging. Pediatr Neurosurg 52 : 80-86, 2017

21. Matsuda R, Coello AF, De Benedictis A, Martinoni M, Duffau H : Awake mapping for resection of cavernous angioma and surrounding gliosis in the left dominant hemisphere: surgical technique and functional results: clinical article. J Neurosurg 117 : 1076-1081, 2012

22. Metting Z, Cerliani L, Rödiger LA, van der Naalt J : Pathophysiological concepts in mild traumatic brain injury: diffusion tensor imaging related to acute perfusion CT imaging. PLoS One 8 : e64461, 2013

23. Min ZG, Niu C, Zhang QL, Zhang M, Qian YC : Optimal factors of diffusion tensor imaging predicting corticospinal tract injury in patients with brain tumors. Korean J Radiol 18 : 844-851, 2017

24. Mormina E, Longo M, Arrigo A, Alafaci C, Tomasello F, Calamuneri A, et al. : MRI tractography of corticospinal tract and arcuate fasciculus in high-grade gliomas performed by constrained spherical deconvolution: qualitative and quantitative analysis. AJNR Am J Neuroradiol 36 : 1853-1858, 2015

25. Nimsky C, Ganslandt O, Fahlbusch R : Implementation of fiber tract navigation. Neurosurgery 61 : 306-317; discussion 317-308, 2007

26. Nimsky C, Ganslandt O, Fahlbusch R : Implementation of fiber tract navigation. Neurosurgery 58 (4 Suppl 2) : ONS-292-ONS-303; discussion ONS-303-ONS-304, 2006

27. Nimsky C, Ganslandt O, Hastreiter P, Wang R, Benner T, Sorensen AG, et al. : Preoperative and intraoperative diffusion tensor imaging-based fiber tracking in glioma surgery. Neurosurgery 61 : 178-185; discussion 186, 2007

28. Orringer DA, Golby A, Jolesz F : Neuronavigation in the surgical management of brain tumors: current and future trends. Expert Rev Med Devices 9 : 491-500, 2012

29. Panigrahi M, Chandrasekhar YB, Vooturi S, Ram GA, Rammohan VS : Surgical resection of insular gliomas and roles of functional magnetic resonance imaging and diffusion tensor imaging tractography-single surgeon experience. World Neurosurg 98 : 587-593, 2017

30. Park ES, Cho YH, Kim JH, Kim SJ, Khang SK, Kim CJ : Frontal transcortical approach in 12 central neurocytomas. Acta Neurochir (Wien) 154 : 1961-1971; discussion 1972, 2012

31. Pierpaoli C, Jezzard P, Basser PJ, Barnett A, Di Chiro G : Diffusion tensor MR imaging of the human brain. Radiology 201 : 637-648, 1996

32. Potts MB, Chang EF, Young WL, Lawton MT; UCSF Brain AVM Study Project : Transsylvian-transinsular approaches to the insula and basal ganglia: operative techniques and results with vascular lesions. Neurosurgery 70 : 824-834; discussion 834, 2012

33. Rosenstock T, Giampiccolo D, Schneider H, Runge SJ, Bährend I, Vajkoczy $P$, et al. : Specific DTI seeding and diffusivity-analysis improve the quality and prognostic value of TMS-based deterministic DTI of the pyramidal tract. Neuroimage Clin 16 : 276-285, 2017
34. Shah PA : Transcranial motor evoked potential monitoring outcome in the high-risk brain and spine surgeries: correlation of clinical and neurophysiological data - an Indian perspective. Ann Indian Acad Neurol $16: 609-613,2013$

35. Shahar T, Rozovski U, Marko NF, Tummala S, Ziu M, Weinberg JS, et al. : Preoperative imaging to predict intraoperative changes in tumorto-corticospinal tract distance: an analysis of 45 cases using high-field intraoperative magnetic resonance imaging. Neurosurgery 75 : 2330, 2014

36. Shanmuganathan K, Zhuo J, Chen HH, Aarabi B, Adams J, Miller C, et al. : Diffusion tensor imaging parameter obtained during acute blunt cervical spinal cord injury in predicting long-term outcome. J Neurotrauma 34 : 2964-2971, 2017

37. Sollmann N, Wildschuetz N, Kelm A, Conway N, Moser T, Bulubas L, et al. : Associations between clinical outcome and navigated transcranial magnetic stimulation characteristics in patients with motor-eloquent brain lesions: a combined navigated transcranial magnetic stimulationdiffusion tensor imaging fiber tracking approach. J Neurosurg 128 : 800-810, 2018

38. Stieltjes B, Kaufmann WE, van Zijl PC, Fredericksen K, Pearlson GD, Solaiyappan $\mathrm{M}$, et al. : Diffusion tensor imaging and axonal tracking in the human brainstem. Neuroimage $14:$ 723-735, 2001

39. Uribe JS, Vale FL : Limited access inferior temporal gyrus approach to mesial basal temporal lobe tumors. J Neurosurg 110 : 137-146, 2009

40. Vassal F, Schneider F, Sontheimer A, Lemaire JJ, Nuti C : Intraoperative visualisation of language fascicles by diffusion tensor imaging-based tractography in glioma surgery. Acta Neurochir (Wien) 155 : 437448, 2013

41. Weiss Lucas C, Tursunova I, Neuschmelting V, Nettekoven C, OrosPeusquens AM, Stoffels $G$, et al. : Functional MRI vs. navigated TMS to optimize M1 seed volume delineation for DTI tractography. A prospective study in patients with brain tumours adjacent to the corticospinal tract. Neuroimage Clin 13 : 297-309, 2016

42. Westin CF, Maier SE, Mamata H, Nabavi A, Jolesz FA, Kikinis R : Processing and visualization for diffusion tensor MRI. Med Image Anal 6 : 93108, 2002

43. Yu CS, Li KC, Xuan Y, Ji XM, Qin W : Diffusion tensor tractography in patients with cerebral tumors: a helpful technique for neurosurgical planning and postoperative assessment. Eur J Radiol 56 : 197-204, 2005

44. Zhang Y, Mao Z, Wei P, Jin Y, Ma L, Zhang J, et al. : Preoperative prediction of location and shape of facial nerve in patients with large vestibular schwannomas using diffusion tensor imaging-based fiber tracking. World Neurosurg $99:$ 70-78, 2017

45. Zhukov VY, Goryaynov SA, Ogurtsova AA, Ageev IS, Protskiy SV, Pronin $I N$, et al. : Diffusion tensor imaging tractography and intraoperative neurophysiological monitoring in surgery of intracranial tumors located near the pyramidal tract. Zh Vopr Neirokhir Im N N Burdenko 80 : 5-18, 2016 